\title{
Interaction of noise disturbances and streamwise streaks
}

\author{
Philipp Schlatter ${ }^{1}$, Luca Brandt ${ }^{1}$ and Rick de Lange ${ }^{2}$ \\ ${ }^{1}$ Linné Flow Centre, KTH Mechanics, Stockholm, Sweden \\ 2 TUe Mechanical Engineering, Eindhoven, The Netherlands \\ pschlatt at mech.kth.se
}

Disturbance evolution in a boundary layer with streamwise streaks and random two- and three-dimensional noise of various amplitudes is studied via numerical simulations. The aim of the present work is to determine the impact of the interaction on the arising flow structures and, eventually, on the location and details of the breakdown to turbulence. It is shown that largescale $2 \mathrm{D}$ noise can be controlled via streaks, whereas the more general 3D noise configuration is prone to premature transition due to increased instability of the introduced streaks. It is interesting to note that the latter transition scenario closely resembles the flow structures found in bypass transition.

A recent theoretical and numerical study by Cossu and Brandt [2] has shown that a substantial stabilisation of a boundary layer subject to essentially two-dimensional disturbances (i.e. Tollmien-Schlichting (TS) waves) can be achieved by a spanwise modulation of the mean flow, i.e. via superimposed streamwise streaks on the laminar Blasius flow. In particular, it has been shown both experimentally via finite-amplitude roughness [3] and later via large-eddy simulation (LES, [5]) that transition to turbulence can effectively by moved to a more downstream position via this essentially passive control mechanism. However, the disturbances considered in the mentioned studies have all had their maximum energy in two-dimensional (spanwise invariant) modes. It is therefore interesting to examine the interaction of streamwise streaks with disturbences of a more general nature, i.e. 2D and 3D random noise at various frequencies and (spanwise) wavenumbers.

The present study uses a spectral numerical method [1] together with LES based on high-order filtering (ADM-RT model [6]), in a similar setup as presented in Ref. [5]. In particular, the computational domain starts at $R e_{x}=$ 32000 extending up to $R e_{x}=590000$. The streaks are introduced at the inlet as optimal disturbances computed from PSE (parabolised stability equations), subsequently evolving nonlinearly inside the domain. Conversely, the noise is forced within the computational domain at $R e_{x}=60000$ by a volume force close to the wall. Two frequency spectra of the noise are shown in Fig. 


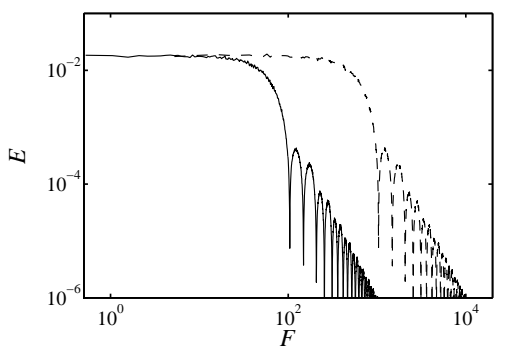

Fig. 1. Frequency spectrum of - large-scale and ----small-scale noise. The maximum spanwise scale based on the local boundary-layer thickness $\delta_{99}$ at the position of forcing $\left(R e_{x}=60000\right)$ for the $3 \mathrm{D}$ noise cases is chosen as $\lambda_{z, \text { max }}=2.25 \delta_{99}$ and $\lambda_{z, \max }=0.75 \delta_{99}$ for the large and small-scale noise, respectively.

1 , specifying the frequencies and spanwise scales denoted "large-scale" and "small-scale" in the following. Note that for all simulations small-amplitude three-dimensional noise is included to enable transition to turbulence.

The amplitude of the noise is determined in such a way that transition to turbulence, i.e. the appearance of a turbulent patch, could be observed in the computational box $\left(R e_{x}<590000\right)$ for the uncontrolled setup (no streaks). The same noise was then used to investigate the interaction of the noise with the streamwise streaks. Depending on the nature of the noise (dimensionality and scales), different amplitudes had to be chosen: two-dimensional, fine-scale noise proved to be more efficient for reaching transition, requiring $u_{\mathrm{rms}, \text { noise }} \approx$ $2.6 \%$. On the other hand, 2D large-scale noise lead to transition only with the significantly larger amplitude of $18.3 \%$. The three-dimensional noise cases lie in between these values.

Directly connected to the different amplitudes required for the noise disturbances in the uncontrolled cases is the predominant transition scenario observed in the flow. Sample visualisations are shown in Figs. 2a) and 3a). It becomes clear that two-dimensional small-scale noise in fact leads to the appearance of spanwise uniform waves, similar to TS-waves, see Fig. 2a). The growth associated to the TS-waves is sufficient to lead to non-linear breakdown within the computational box even for the mentioned low amplitudes. On the other hand, large-scale 2D noise does not excite any growing instability in the boundary layer at the considered Reynolds numbers due to the low frequency $F<80$ and the two-dimensionality of the disturbances (i.e. no liftup mechanism). Therefore, intermittent turbulent spots are directly triggered at the forcing position, which then grow downstream (not shown).

For the cases with three-dimensional noise, the dominant instability mechanism is non-modal growth based on the lift-up mechanism, generating to streamwise streaks. Again, a dependence of the arising flow structures on the scales of the disturbances is observed: Fine scales tend to decay quickly, whereas larger scales lead to a flow with significant streamwise streaks, which then might get unstable developing a growing wave packet [4] and finally 
a)

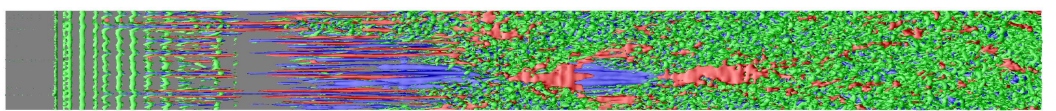

b)

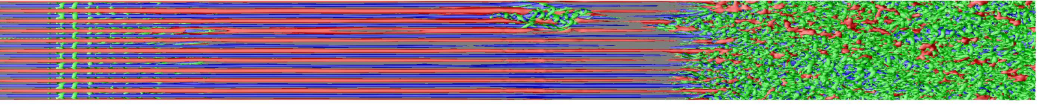

Fig. 2. Top view of the three-dimensional flow structures for cases with $2 \mathrm{D}$, fine-scale noise. a) no streaks. b) Streaks with amplitude 19\%. Light isocontours represent the $\lambda_{2}$ vortex-identification criterion, dark and light grey isocontours are positive and negative disturbance velocity. Flow from left to right.

a)

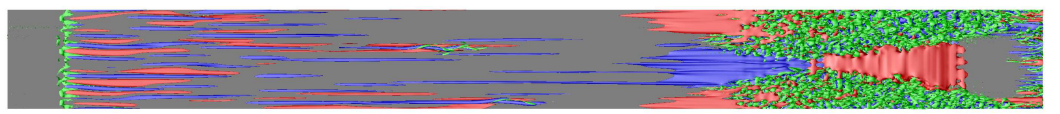

b)

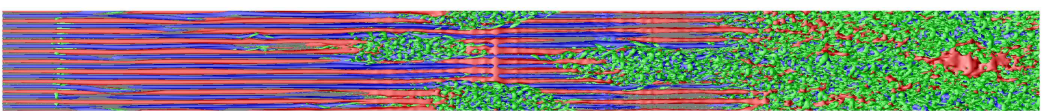

Fig. 3. Top view of the three-dimensional flow structures for cases with $3 \mathrm{D}$ largescale noise, same isocontour levels and colours as in Fig. 2. a) no streaks. b) streaks with amplitude $19 \%$.

break down into triangle-shaped turbulent spots as seen in Fig. 3a). The flow structures appearing in these cases are similar to observations in bypass transition induced by ambient free-stream turbulence. Note that in the presence of strong streaks - irrespective of whether they are actively introduced in the flow or naturally arise due to e.g. free-stream turbulence, the turbulent spots do not feature a clear triangular shape.

The efficiency of the imposed streamwise streaks to damp the amplification of disturbances in the boundary layer is naturally strongly dependent on the nature of these disturbances and the respective transition scenario. The damping abilities of streaks has been demonstrated for cases with dominant two-dimensional waves, see the references mentioned above $[2,3,5]$. Consequently, for the case which leads to TS-wave dominated transition (2D small-scale noise), transition delay can be observed, see Fig. 2b). Quantitative data is given in Fig. 4a) showing the disturbance growth inside the boundary layer. However, as opposed to cases with clean TS-waves (for example $F=120$ as in Ref. [5]) no complete stabilisation of the boundary layer can be achieved; intermittent turbulent spots are appearing further downstream as an instability of the strong streaks.

On the other hand, three-dimensional noise produces a change of the disturbance growth mechanism in the boundary layer from modal (TS-waves) to non-modal (lift-up, streaks). The spanwise periodic base flow created by forcing large-amplitude streaks does not lead to any transition delay. To the contrary, turbulent spots can be observed more frequently and further upstream, see Fig. 3b). As for the uncontrolled case, the transition scenario is a secondary instability of the streak, characterised by a growing wave packet 

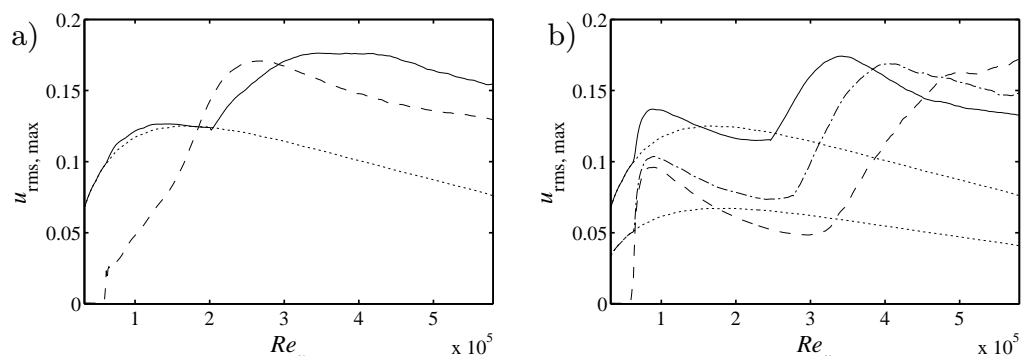

Fig. 4. a) Maximum streamwise fluctuations $u_{\mathrm{rms}, \max }$ for $\stackrel{\text { Re }}{\mathrm{k}}$ streaks $(19 \%)$ and small-scale 2D noise (amplitude 0.3\%), ----only noise, no streaks, ….... undisturbed streaks. b) $u_{\mathrm{rms}, \max }$ for streaks $(-19 \%,-\cdot-10 \%)$ and largescale 3D noise, ----only noise (amplitude 0.9\%), no streaks, $\cdots \cdots \cdot$ undisturbed streaks.

riding on the streak [4]. However, due to the larger amplitude of the streaks, this breakdown occurs further upstream. In Fig. 4b) the growth of $u_{\text {rms }}$ inside the boundary layer is shown; the breakdown location is clearly moving upstream for larger amplitudes of the introduced streaks.

It can therefore be concluded that the passive control mechanism based on a spanwise modulated base flow and the subsequent damping of the growth of TS-waves [2] is very efficient for transition scenarios based on the modal growth of essentially two-dimensional disturbances. On the other hand, if transition is induced by (non-modal) growth related to bypass transition, then the addition of a strong base-flow modulation might lead to premature transition. Similar results have been obtained for transition induced by free-stream turbulence (not shown), for which as expected no transition delay could be observed.

\section{References}

1. M. Chevalier, P. Schlatter, A. Lundbladh and D. S. Henningson. SImson - A Pseudo-Spectral Solver for Incompressible Boundary Layer Flows. Tech. Rep., TRITA-MEK 2007:07, KTH Mechanics, Stockholm, Sweden, 2007.

2. C. Cossu and L. Brandt. Stabilization of Tollmien-Schlichting waves by finite amplitude optimal streaks in the Blasius boundary layer. Phys. Fluids, 14(8):L57L60, 2002.

3. J. H. M. Fransson, A. Talamelli, L. Brandt and C. Cossu. Delaying transition to turbulence by a passive mechanism. Phys. Rev. Lett., 96(064501):1-4, 2006.

4. P. Schlatter, L. Brandt, H. C. de Lange and D. S. Henningson. On streak breakdown in bypass transition. Phys. Fluids, 20(101505):1-15, 2008.

5. P. Schlatter, H. C. de Lange and L. Brandt. Numerical study of the stabilisation of Tollmien-Schlichting waves by finite amplitude streaks. In Turbulence and Shear Flow Phenomena 5, edited by R. Friedrich et al., 849-854, 2007.

6. P. Schlatter, S. Stolz and L. Kleiser. LES of transitional flows using the approxiamte deconvolution model. Int. J. Heat Fluid Flow, 25(3):549-558, 2004. 